\title{
Aerosol Delivery in Ventilated Newborn Pigs: An MRI Evaluation
}

\author{
BEENA G. SOOD, YIMIN SHEN, ZAHID LATIF, XINGUANG CHEN, JODY SHARP, JALADHAR NEELAVALLI, \\ APARNA JOSHI, THOMAS L. SLOVIS, AND E. M. HAACKE
}

\author{
Department of Pediatrics [B.G.S., X.C., J.S.], Department of Radiology [Y.S., Z.L., A.J., T.L.S., E.M.H.], Department of Biomedical \\ Engineering [J.N.], Wayne State University, Detroit, MI 48201
}

\begin{abstract}
Pulmonary deposition of inhaled drugs in ventilated neonates has not been studied in vivo. The objective of this study was to evaluate pulmonary delivery of gadopentetate dimeglumine $(\mathrm{Gd}-$ DTPA) following nebulization in ventilated piglets using magnetic resonance imaging. Seven ventilated piglets $(5 \pm 2 \mathrm{~d}$ old, weight $1.8 \pm 0.5 \mathrm{~kg}$ ) were scanned in the Bruker/Siemens $4 \mathrm{~T}$ magnetic resonance scanner using T1 weighted spin-echo sequence. Aerosols of Gd-DTPA were generated continuously using the MiniHeart jet nebulizer. Breath-hold coronal images were obtained before and every $10 \mathrm{~min}$ during aerosolized Gd-DTPA for $90 \mathrm{~min}$. Signal intensity (SI) changes over the lungs, kidneys, liver, skeletal muscle, and heart were evaluated. A significant increase in SI was observed in the lungs, kidney, and liver at 10, 20, and 40 min respectively after start of aerosol. At the end of $90 \mathrm{~min}$, the SI increased by $95 \%, 101 \%$, and $426 \%$ over the right lung, left lung, and kidney, respectively. A much smaller increase in SI was observed over the liver. In conclusion, we have demonstrated effective pulmonary aerosol delivery within $10 \mathrm{~min}$ of contrast nebulization in ventilated piglets. Contrast visualization in the kidneys within $20 \mathrm{~min}$ of aerosol initiation reflects alveolar absorption, glomerular filtration and renal concentration. (Pediatr Res 64: 159-164, 2008)
\end{abstract}

$\mathrm{N}^{\mathrm{en}}$ eonatal hypoxemic respiratory failure (NHRF) is associated with potentially reversible pulmonary hypertension that causes right-to-left shunting and profound hypoxemia. Inhaled nitric oxide, a selective pulmonary vasodilator, has revolutionized the treatment of NHRF (1). However, lack of sustained improvement in $30-46 \%$ of infants and the need for specialized delivery systems make the treatment expensive and limit availability. Several investigators have explored the use of aerosolized prostaglandin $\mathrm{E}_{1}\left(\mathrm{PGE}_{1}\right)$ as a selective pulmonary vasodilator in patients with respiratory failure to improve oxygenation because of its selective action not only on the pulmonary circulation, but also on well-ventilated lung units (2-5). We have previously described the safety and feasibility of inhaled $\mathrm{PGE}_{1}\left(\mathrm{IPGE}_{1}\right)$ in NHRF in a phase I/II unblinded clinical trial (5). We have also reported the stability and emitted dose of IPGE $_{1}$ following jet nebulization in a neonatal ventilator circuit and predicted primarily alveolar

Received November 19, 2007; accepted March 14, 2008.

Correspondence: Beena G. Sood, M.D., M.S., Department of Pediatrics, Children's Hospital of Michigan, 3901 Beaubien Blvd., 4H42, Detroit, MI 48201; e-mail: bsood@med.wayne.edu

This research was funded in part by Grant 1 K23 HD41423-01 from the National Institute of Child Health and Human Development and the Children's Research Center of Michigan. deposition based on the aerosol particle size distribution (6). Alveolar deposition is important for optimal drug effect. Although it is recognized that aerosolized drug delivery is more efficient in spontaneously breathing subjects compared with those undergoing assisted ventilation and in adults compared with children and neonates, the deposition pattern of inhaled drugs in ventilated neonates remains largely unknown because of ethical concerns in this age group $(7,8)$. Several investigators have reported the feasibility of performing magnetic resonance (MR) ventilation imaging in animal models and human adults with aerosolized Gadopentetate dimeglumine (Gd-DTPA) as a contrast agent $(9-17)$. The present study was designed to assess aerosol delivery in neonatal pigs undergoing mechanical ventilation using functional MR imaging (MRI) with aerosolized gadopentetate dimeglumine (Gd-DTPA) as a surrogate.

\section{MATERIALS AND METHODS}

Subjects. The study was performed on nine healthy domestic piglets (2-6 d old) from a specific pathogen free litter after approval by the Institutional Animal Care and Use Committee. All animals received care in compliance with the National Institutes of Health guidelines.

Animal preparation. Animals were intubated oro-tracheally after intramuscular premedication with midazolam and ketamine. The cephalic vein was catheterized percutaneously for vascular access. Anesthesia was maintained by i.v. infusion of ketamine, midazolam, and fentanyl titrated to the level of anesthesia as assessed by monitoring heart rate, blood pressure, and response to painful stimuli. Maintenance fluids consisting of dextrose-saline were administered. Body temperature was maintained at $38-39^{\circ} \mathrm{C}$. Baseline measurements of blood gas parameters were obtained followed by MRI.

Mechanical ventilation. Positive pressure ventilation was provided manually at a rate of $15 \mathrm{bpm}$ and peak inspiratory pressure of $18 \mathrm{~cm} \mathrm{H}_{2} \mathrm{O}$ using room air by trained research staff during the MRI protocol. The ventilator circuit used is similar to the one used for hospitalized neonates undergoing mechanical ventilation in the MRI suite. Oxygen saturations were monitored using a pulse oximeter. The gas delivered into the circuit was neither heated nor humidified. All experiments were carried out at room temperature.

Administration of continuous aerosol. The low flow MiniHeart jet nebulizer (Westmed Inc., Lakewood, Co) was used to generate continuous aerosols as described previously (5). The nebulizer was placed in the inspiratory limb of the ventilator circuit close to the ETT. The oxygen flow through the jet nebulizer was set at 2 LPM. Because oxygen is known to be a paramagnetic contrast agent, the nebulizer was placed in the nebulizer circuit from the beginning of the experiment to assess baseline conditions. At the start of aerosol therapy, the nebulizer chamber was primed with $4 \mathrm{~mL}$ of Gd-DTPA $(0.5 \mathrm{~mol} / \mathrm{L})$ followed by continuous delivery into the nebulizer chamber at a rate of $8 \mathrm{~mL} / \mathrm{h}$. The MiniHeart nebulizer generates aerosols with a mean particle size of $2-3 \mu \mathrm{m}$.

\footnotetext{
Abbreviations: Gd-DTPA, gadopentetate dimeglumine; MR, magnetic resonance; NHRF, neonatal hypoxemic respiratory failure; $\mathbf{P G E}_{\mathbf{1}}$, prostaglandin $\mathrm{E}_{1}$; SI, signal intensity
} 
MR imaging. Anesthetized term newborn piglets undergoing manual positive pressure ventilation were scanned in the Bruker 4T MR scanner in the supine position using T1 weighted spin-echo sequence with a head coil used for excitation and reception. The first scan was performed before the administration of aerosolized Gd-DTPA to assess baseline conditions. Next, GdDTPA was infused into the nebulizer chamber without moving the animal from its original position inside the tomograph. Serial images at the same anatomic level were obtained for 90 min during ventilation with aerosolized Gd-DTPA. To reduce motion artifacts from breathing, respiratory gating was accomplished by data acquisition in the inspiratory phase (18 s) during a breath hold. Respiratory-gated coronal images were obtained before and every 10 min during aerosolized Gd. The MRI parameters included: TE of $12 \mathrm{ms,}$ TR of $200 \mathrm{~ms}$, one NEX (number of excitations), field of view of $250 \times 125$ $\mathrm{mm}^{2}$, slice thickness of $5 \mathrm{~mm}$ with nine continuous slices acquired interleaved, base resolution 256, and phase resolution of 96 then interpolated to $512 \times 256$, phase partial Fourier 6/8. The reception bandwidth was $150 \mathrm{~Hz}$. Signal intensity changes over corresponding regions of the lungs, liver, kidneys, skeletal muscle, and heart were monitored over time to assess Gd-DTPA deposition.

MR images were analyzed using our internally developed MR signal processing in NMR (SPIN) software that is written in Visual $\mathrm{C}++$ for the Microsoft Windows platform (18). Signal intensities were measured in defined regions of interest before and at each time point after administration of aerosolized agent $(0,10,20,30,40,50,60,70,80$, and $90 \mathrm{~min})$. Regions of interest were defined in lungs, liver, heart, kidney, and skeletal muscle. Regions were chosen to contain a minimum of 100 pixels. The percent enhancement of signal intensity (SI) over baseline was calculated by means of the following formula: $\left(\left[\mathrm{SI}_{\text {postcontrast }}-\mathrm{SI}_{\text {precontrast }}\right] / \mathrm{SI}_{\text {precontrast }}\right) \times 100$. Subtraction images were generated as the difference between the Gd-DTPAenhanced images and baseline images.

Tissue concentration of $\mathrm{Gd}$ was extracted from the ratio of the signal intensity as a function of time, $s(t)$, to the signal intensity at baseline, $s 0$. Signal intensity as a function of time is proportional to $[1-\exp (-\mathrm{TR} / \mathrm{T} 1(\mathrm{t}))]$. The ratio $\mathrm{s}(\mathrm{t}) / \mathrm{s} 0$ can thus be calculated directly. To convert this ratio to concentration requires a knowledge of $\mathrm{T} 1(0)$ in the equation $1 / \mathrm{T} 1(\mathrm{t})=$ $1 / \mathrm{T} 1(0)+\alpha \times \mathrm{c}(\mathrm{t})$, where $\alpha$ is approximately $5(\mathrm{mM} \mathrm{sec})^{-1}, \mathrm{c}(\mathrm{t})$ is the tissue concentration of $\mathrm{Gd}$ as a function of time, and $\mathrm{T} 1(\mathrm{t})$ is calculated by $\mathrm{T} 1(\mathrm{t})=$ $-\mathrm{TR} / \ln \{1-[1-\exp (-\mathrm{TR} / \mathrm{T} 1(0))] \mathrm{s}(\mathrm{t}) / \mathrm{s} 0)\}$ for spin echo. Because T1(0) is not well characterized at $4 \mathrm{~T}$, a range of $\mathrm{T} 1(0)$ values for the lung $(0.8,1.2$, and $1.6 \mathrm{~s})$ and kidney $(1.4,1.6$, and $1.8 \mathrm{~s})$ were used to estimate a range of concentrations.

Euthanasia. At the end of the experimental protocol, the piglets were euthanized by i.v. overdose of pentobarbital $(90 \mathrm{mg} / \mathrm{kg})$ and bilateral pneumothoraces.

Statistical analyses. Descriptive statistics were used to summarize sample characteristics. Sample means and standard errors of the detected signal intensities were calculated for lungs, kidney, liver, heart, and skeletal muscle at each of the 10 time points before the administration of the contrast agent till the end of the experiment. The mean signal intensities (standard errors) were plotted against time to delineate time trends. Linear mixed models were used to assess the increases in detected signal intensities over time (19). Although the detected signal intensities for the left lungs appeared more variable than those from the right lungs, the difference was not statistically significant. Data from the two lungs were thus combined in analysis. Similarly, average data for the kidneys was used in the analysis. Statistical significance was defined as a $p$ value of less than 0.05 . Statistical analyses were performed using the SPSS statistical package, version 15.0.1 (SPSS Inc., Chicago, IL) and SAS/ STAT software, Version [9.1.3] (SAS Institute Inc., Cary, NC).

\section{RESULTS}

Nine intubated term newborn piglets underwent MR scans. Two pigs died during transport before contrast administration (tension pneumothorax $=1$; massive pulmonary hemorrhage $=1$ ). The remaining seven pigs received aerosolized Gd. Only data for the seven pigs that received aerosolized Gd-DTPA are presented. The mean \pm SE age and weight of the piglets was $5 \pm 2 \mathrm{~d}$ and $1.8 \pm 0.5 \mathrm{~kg}$, respectively.

On visual inspection of the MR scans, enhancement of the collecting system of the kidneys was dramatic and observed in all animals at $30 \mathrm{~min}$ (Fig. 1). Figure 2 shows marked enhancement of the left lung at the end of 90 min that is better

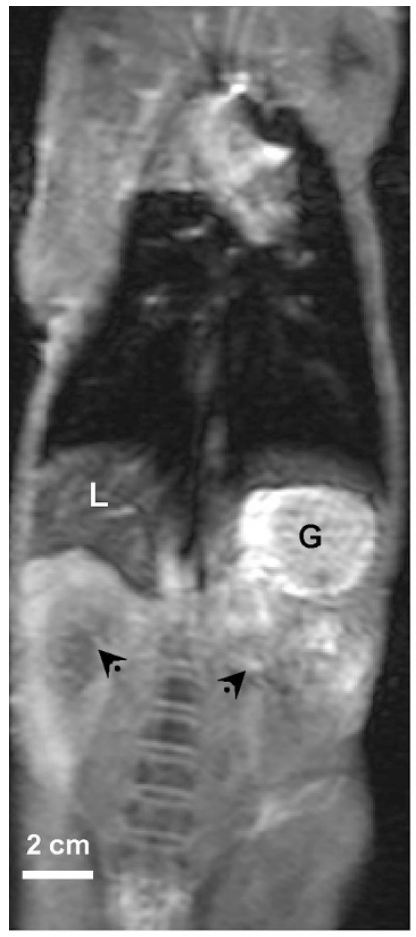

\section{Before Gd}

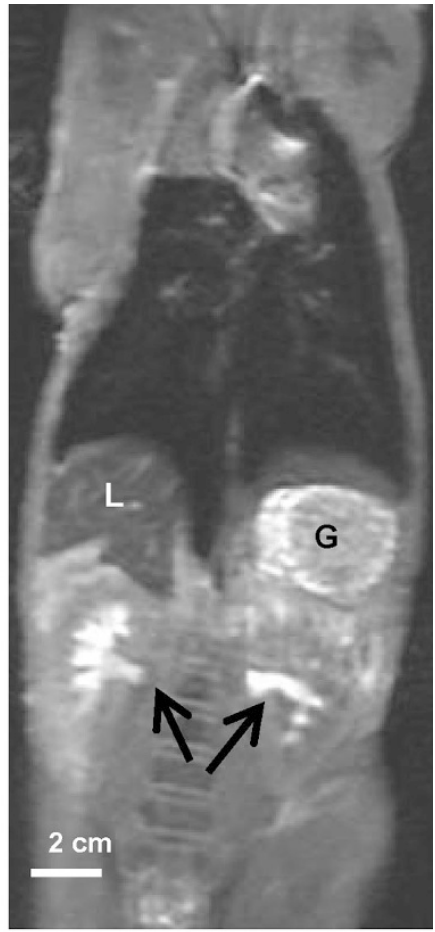

After 30min of Gd
Figure 1. SI changes on T1-weighted images in healthy ventilated piglets after inhalation of Gd-DTPA. (A) At baseline, before contrast administration, both lungs have low SI (21 for left lung and 14 for right lung) and the renal collecting system appears dark (arrowheads). The liver (L) and gastric contents (G) are indicated. (B) 30 min after aerosolized Gd-DTPA inhalation, both lungs enhance homogeneously (SI = 37 for left lung, 26 for right lung) and contrast is visualized in the renal collecting system (arrows). The SI over the liver and gastric contents is unchanged.

visualized on the subtraction image obtained by subtracting the baseline image from the $90 \mathrm{~min}$ postcontrast image.

The percent increase in lung SI in healthy ventilated piglets after administration of aerosolized Gd is shown in Figs. 3 (first $20 \mathrm{~min}$ ) and 4 (over entire duration of study). The baseline SI before administration of aerosolized $\mathrm{Gd}$ was assigned a value of 0 . After inhalation of aerosolized Gd-DTPA, the detected signal intensity in lungs increased significantly $(p<0.05)$ above baseline as early as $10 \mathrm{~min}$ after onset of aerosol for both lungs (mean \pm SE increase in SI for left lung $9 \pm 3 \%$, $p<0.05$; right lung $13.5 \pm 2 \%, p<0.01$; average for both lungs $11.3 \pm 2 \%, p<0.01$ ) (Fig. 1). There was a significant increase in SI over both lungs with time on longitudinal data analysis using proc mixed (estimate $=1.4, \mathrm{SE}=0.4, p<$ 0.05 ) (Fig. 2). At the end of $90 \mathrm{~min}$, the signal intensity in the right and left lung increased by (mean \pm SD) $95 \pm 40$ and $101 \pm 47 \%$, respectively, with an average increase of $98 \pm$ $38 \%$. The change in SI over the lungs as a function of time could be described by the following linear regression equation: Change in SI $=1.2 \times$ Time $(\min )-10\left(r^{2}=0.9\right)$.

Significant increase in SI over the kidneys was first detected 20 min after the onset of aerosol therapy $(31 \pm 11 \%, p<$ 0.05) (Fig. 1). There was a significant increase of SI over the kidneys with time (estimate 6.1, $\mathrm{SE}=1.0, p<0.01$ ) (Fig. 2); at the end of $90 \mathrm{~min}$, the signal intensity in the collecting 

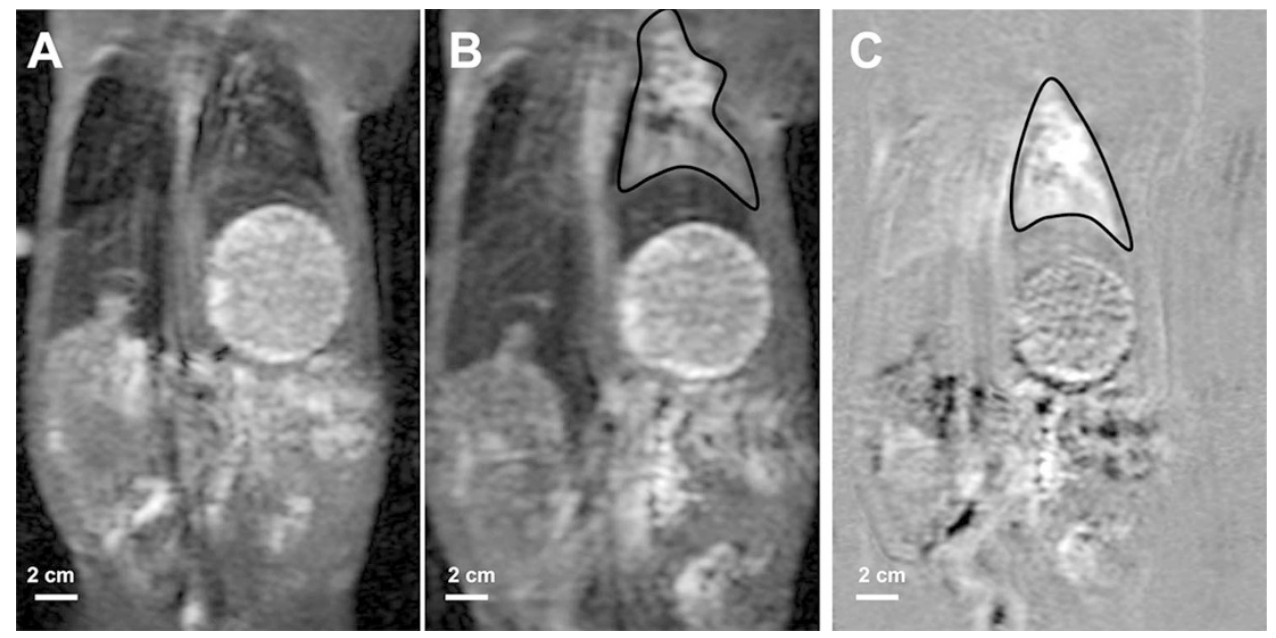

Figure 2. Significant increase in SI over left lung (outlined) at $90 \mathrm{~min}(B)$ compared with baseline $(A)$. This enhancement is better visualized on the subtraction image (C). Change in signal intensity over the right lung is not as marked in these scans.

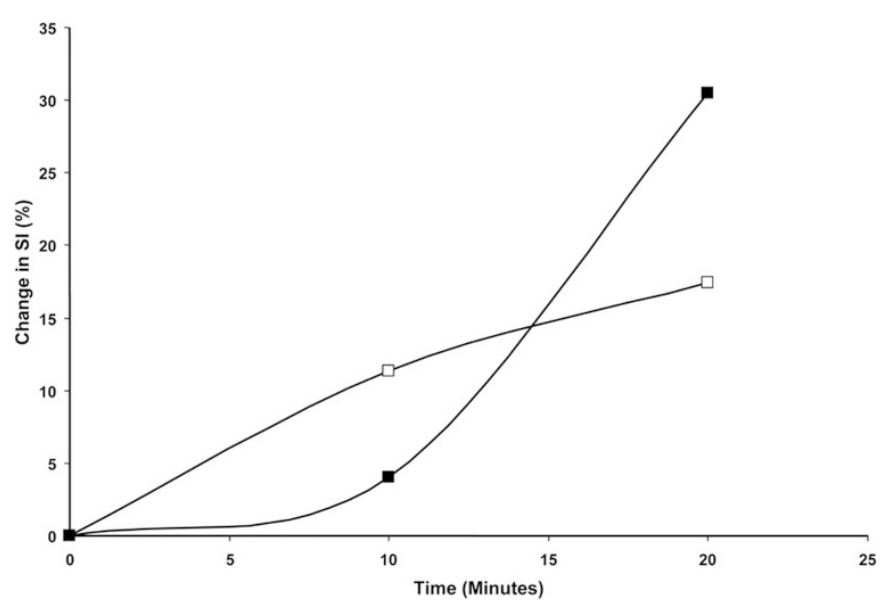

Figure 3. Changes in SI over lungs ( $\square$ ) and kidneys ( $\square$ ) during first $20 \mathrm{~min}$ of aerosol administration.

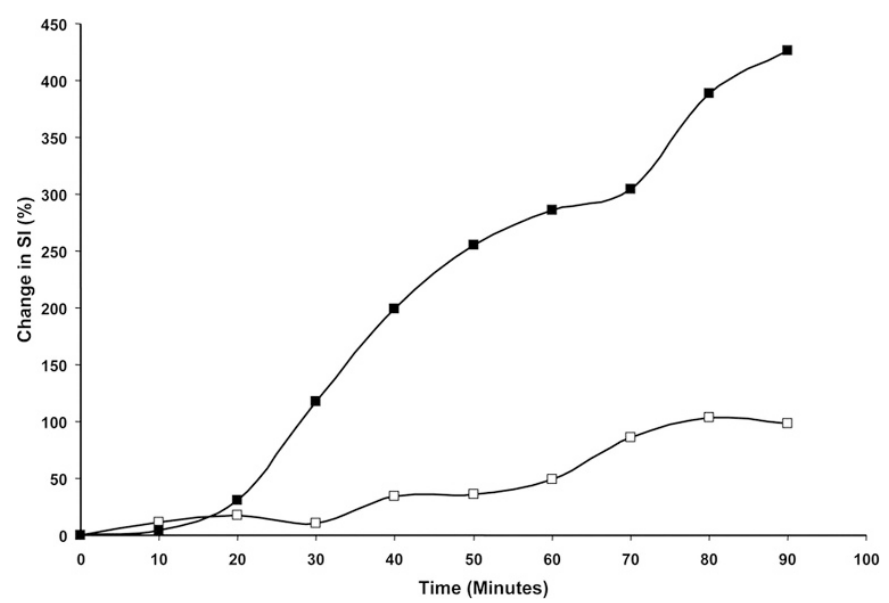

Figure 4. Changes in SI over lungs $(\square)$ and kidneys ( $\square$ ) during aerosol administration over $90 \mathrm{~min}$.

system of the kidneys was $426 \pm 60 \%$ above baseline. The change in SI over the kidneys as a function of time could be described by the following linear regression equation: Change in SI $=5.1 \times$ Time $(\min )-29\left(r^{2}=0.98\right)$.

Although there was a significant increase in signal intensity over the liver and skeletal muscle with duration of aerosol therapy (estimate $0.1, \mathrm{SE}=0.05, p<0.05$ ), the absolute

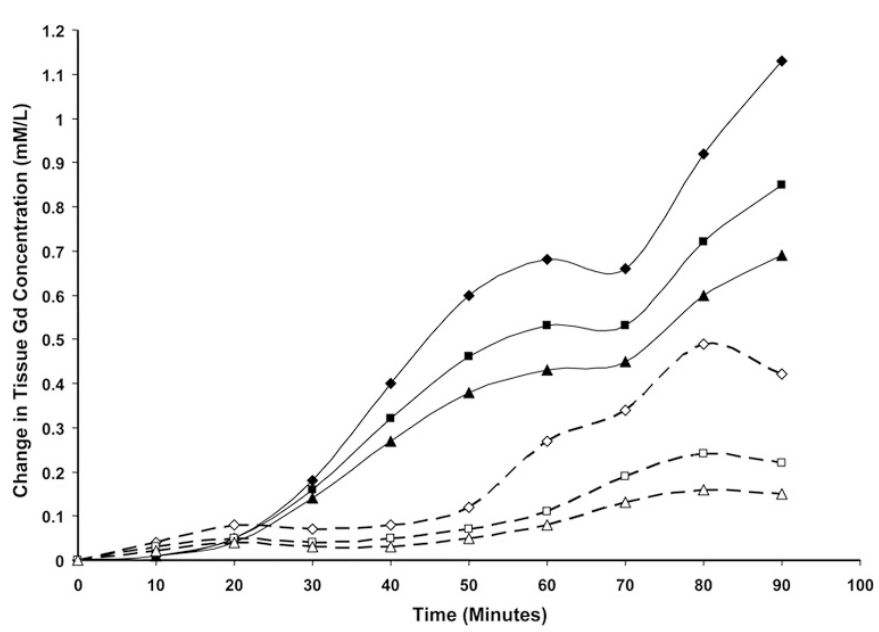

Figure 5. Tissue concentration of Gd in kidney (solid lines) and lung (interrupted lines) over time as calculated from the changes in signal intensity. $\mathrm{T} 1(0)$ values used were $0.8 \mathrm{~s}(\diamond), 1.2 \mathrm{~s}(\square)$, and $1.6 \mathrm{~s}(\triangle)$ for lung; and $1.4 \mathrm{~s}$ $(\diamond), 1.6 \mathrm{~s}(\mathbf{\square})$, and $1.8 \mathrm{~s}(\mathbf{\Delta})$ for kidney.

increase in signal intensity was much lower than over the kidneys and lungs and was first observed 40 min after start of aerosol therapy. At the end of $90 \mathrm{~min}$, the increase in SI was over the liver and skeletal muscle was $14 \pm 2 \%$ and $16+6 \%$ above baseline, respectively. The change in SI over the heart was insignificant over the course of the experiment.

Tissue concentrations of $\mathrm{Gd}$ in the lung and kidney for different values of $\mathrm{T} 1(0)$ are shown in Fig. 5. The lung concentrations were higher than the kidney concentrations in the first $20 \mathrm{~min}$. The kidney concentration at $90 \mathrm{~min}(0.69-$ $1.13 \mathrm{mM}$ ) was much higher than the lung $\mathrm{Gd}$ concentration at 90 min $(0.15-0.42 \mathrm{mM})$. There was a significant positive correlation between the tissue concentration of Gd in the lungs and kidneys (correlation coefficient $=0.91$, Fig. 6).

\section{DISCUSSION}

We have successfully demonstrated effective pulmonary aerosol deposition in mechanically ventilated neonatal pigs using MRI with aerosolized Gd-DTPA. Although aerosolized Gd-DTPA has been used to define regional lung ventilation in healthy animals, experimental disease models, and human 


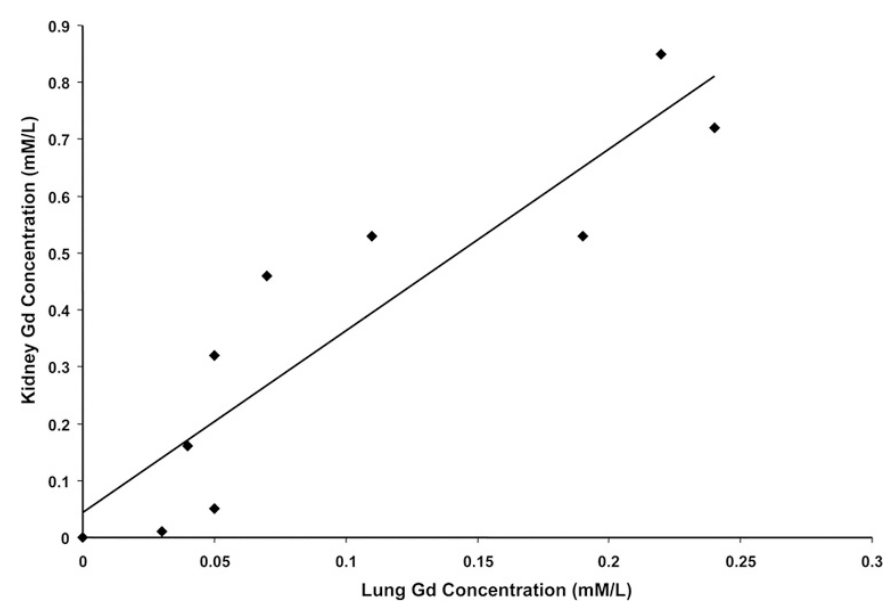

Figure 6. Correlation of Gd concentrations in lung and kidney over time. A $\mathrm{T} 1(0)$ of $1.2 \mathrm{~s}$ was used for lung and $1.6 \mathrm{~s}$ for kidney. The correlation coefficient is 0.91 .

adults, this is the first report of the use of aerosolized GdDTPA to evaluate pulmonary drug delivery in a neonatal animal model undergoing mechanical ventilation (9-17). Neonatal pigs undergoing mechanical ventilation were chosen, as it is known that aerosol drug delivery is decreased in ventilated subjects as well as in infants and children. Pulmonary deposition was demonstrated as early as 10 min following onset of aerosol therapy indicating successful delivery further supported by the detection of increased SI over the kidneys $20 \mathrm{~min}$ after aerosol initiation. MR images were obtained every $10 \mathrm{~min}$ for $90 \mathrm{~min}$ allowing assessment of SI changes over time. Comparison of SI changes over different organs permitted assessment of drug disposition over time. Tissue Gd concentration in lungs and kidneys was quantified from the signal intensity data.

Pulmonary aerosol deposition has been evaluated by theoretical in vitro models, pharmacokinetic studies and pulmonary imaging in animals and humans $(7,8,20-25)$. Theoretical models and pharmacokinetic studies cannot directly demonstrate the site of aerosol deposition; in the latter, it is inferred that plasma and urine levels reflect lower respiratory tract deposition (8). Functional imaging of the lung may allow determination of site of aerosol delivery. Gamma scintigraphy, the gold standard for functional imaging studies, is limited by poor spatial and temporal resolution and radiation exposure (22). Single photon emission computed tomography and positron emission tomography, three-dimensional lung imaging techniques that allow detailed assessment of regional deposition have their limitations. Single photon emission computed tomography data has to be transformed to conceptual lung models that need to be validated. Positron emission tomography is not widely used because of the short half-life of the unstable positron-emitting radioisotopes necessitating an on-site accelerator for production. In contrast, MRI does not rely on ionizing radiation and permits ventilation imaging of the lungs with high spatial and temporal resolution using paramagnetic contrast agents. However, MRI of the lung parenchyma is problematic because of relatively low number of hydrogen protons, cardiac and respiratory motion, and susceptibility effects induced by multiple air and tissue interfaces $(10,26,27)$. These problems can be overcome by the use of pulse sequences with a very short echo time (TE), breath hold imaging, and cardiac gating. Several investigators have reported the feasibility of performing MR ventilation imaging in animal models and human adults with aerosolized gadolinium (Gd)-DTPA as a contrast agent (9-17). Because of imaging with this T1-shortening contrast agent, a high SI can be generated within the pulmonary air spaces. The highly soluble droplets dissolve rapidly and are absorbed into the blood by rapid diffusion across pulmonary epithelium and capillary endothelium. Once the contrast agent is absorbed from the lung into the plasma, the pharmacokinetics are similar to those of a compound administered intravenously. Calculated half-life for pulmonary clearance is approximately $2 \mathrm{~h}$, with completion of urinary elimination by $30 \mathrm{~h}$. Additional advantages of Gd-DTPA aerosol include its wide availability, comparatively low cost, ability to use with existing MR systems without modification, and safety. The present study was designed to assess aerosol delivery in neonatal pigs undergoing mechanical ventilation using functional MRI with aerosolized Gd-DTPA as a surrogate.

We chose the neonatal pig as a model for this in vivo study as the functional thoracic anatomy, lung morphology, pulmonary vasculature, and postnatal development of the pig is close to that of the human being $(28,29)$. In this study, there was a significant increase in the SI over the lungs within $10 \mathrm{~min}$ of aerosol administration reflecting early effective aerosol delivery to the lungs. There was progressive increase in SI over time. These results are comparable to those reported in ventilated animal and human adults in whom variable increases in SI over the lungs have been reported at 10-30 min (ranging from 22 to $216 \%)(12-17,30)$. The greater variability in SI changes over the left lung compared with the right reflects difficulty in accurately measuring SI because a large portion of the left hemithorax is occupied by the heart. To determine whether the increase in SI over the lungs reflected presence of contrast agent in the interstitial fluid or blood vessels, we determined the change in SI over vascular organs like the liver, skeletal muscle, and heart. Although there was a significant increase in SI over the liver and skeletal muscle over time, the SI increase was delayed and smaller in magnitude compared with the lungs suggesting a low concentration in circulating plasma. This implies that SI changes in the lungs reflect the presence of contrast in the interstitial compartment. Montgomery et al. (31) have previously shown that aerosolized Gd-DTPA selectively enhances the extravascularextracellular water spaces of the lung. Significant increase in SI over the kidneys was evident 10 min after the increase in lungs reflecting extensive alveolar absorption; thereafter, the SI increased rapidly over the kidneys, surpassing the SI increase over the lungs. This can be explained by a combination of rapid clearance from the lung, prompt elimination and concentration by the kidneys, and better visualization of a solid organ by MRI. Tissue concentrations of Gd in the lung and kidney were characterized by higher values for the lungs in the first 20 min followed by higher kidney concentrations subsequently. There was a significant positive correlation 
between tissue Gd concentration in lungs and kidneys. This is to be expected if the contrast agent is successfully getting into the blood from the alveoli and is being filtered and concentrated in the kidneys.

This study describes for the first time effective pulmonary drug delivery and distribution following jet nebulization in an in vivo neonatal animal model using functional MRI. However, despite the important findings of this study, there are limitations. First, we used a surrogate agent to determine pulmonary deposition. Aerosol particle size depends upon the physicochemical properties of the agent, nebulizer, and patient related factors. Both PGE $_{1}$ and Gd-DTPA are water-soluble compounds. We have previously reported the physicochemical properties of $\mathrm{PGE}_{1}$ and the aerosol particle size distribution (6). In this report, we have used aerosolized Gd-DTPA as a paramagnetic contrast agent. Physicochemical properties and aerosol particle size have been previously characterized for Gd-DTPA $(11,12)$. The aerosol particle size for both $\mathrm{PGE}_{1}$ and Gd-DTPA favors alveolar deposition; therefore, these results predict pulmonary drug deposition for $\mathrm{PGE}_{1}$ following jet nebulization in a neonatal ventilator circuit. Second, inhaled molecular oxygen has been shown to be an effective MR contrast agent in MR ventilation imaging because of its paramagnetic properties $(27,32,33)$. Although animals in this study received the same flow rate of oxygen during baseline and aerosolized Gd-DTPA administration to avoid the confounding effect of oxygen on SI changes, it is possible that enhancement of signal intensity after administration of aerosolized Gd-DTPA may be attenuated. To address this confounding effect, scans acquired before inhalation of Gd-DTPA were subtracted form those obtained after inhalation of Gd-DTPA (30). Third, although we have demonstrated effective pulmonary deposition, we have not quantified the actual percent of aerosol delivered. Future mass balance studies involving a closed system with collection of exhaled gas and urinary output and complex mathematical models will permit quantification of aerosolized drug delivery. Fourth, in the present study, the animals were manually ventilated without positive end-expiratory pressure using a modified ventilator circuit with extremely long inspiratory tubing and nebulizer close to the ETT. Despite the fact that these factors would have decreased aerosol delivery to the animal, we were able to demonstrate effective pulmonary aerosol delivery. Although precautions were taken to be consistent in delivering breaths of similar duration and inspiratory pressure, an MR compatible mechanical ventilator may have allowed greater consistency in ventilation thus decreasing regional SI differences (12). The subjects of this study were heavily sedated to avoid motion artifacts. Once the animal was placed in the scanner, no changes were made in the position to avoid degradation of image quality during serial scans. However, other factors may have contributed to degradation of image quality including misregistration between serial images because of relatively long time intervals between imaging and cardiac motion. This may account for the relatively large variation in the lung enhancement observed. The low echo time of $12 \mathrm{~ms}$ used in these experiments along with the maximum concentrations of 1-2 mM limits the T2 effect to less than 5-10\% in the kidney.
For concentrations on the order of $0.1-0.2 \mathrm{mM}$, this effect reduces to less than $1 \%$.

In conclusion, our study shows the feasibility of serial MRI assessment of pulmonary drug delivery following administration of aerosolized medications. We have demonstrated effective pulmonary aerosol delivery within $10 \mathrm{~min}$ of contrast nebulization in ventilated piglets. Contrast visualization in the kidneys within 20 min of aerosol initiation reflects extensive alveolar absorption and renal concentration. Future studies are needed to quantify efficiency of aerosolized drug delivery in an in vivo closed system.

\section{REFERENCES}

1. NINOS 1997 Inhaled nitric oxide in full-term and nearly full-term infants with hypoxic respiratory failure. The neonatal inhaled nitric oxide study group. $\mathrm{N}$ Engl J Med 336:597-604

2. Walmrath D, Schermuly R, Pilch J, Grimminger F, Seeger W 1997 Effects of inhaled versus intravenous vasodilators in experimental pulmonary hypertension. Eur Respir J 10:1084-1092

3. Meyer J, Theilmeier G, Van Aken H, Bone HG, Busse H, Waurick R, Hinder F, Booke M 1998 Inhaled prostaglandin E1 for treatment of acute lung injury in severe multiple organ failure. Anesth Analg 86:753-758

4. Putensen C, Hormann C, Kleinsasser A, Putensen-Himmer G 1998 Cardiopulmonary effects of aerosolized prostaglandin E1 and nitric oxide inhalation in patients with acute respiratory distress syndrome. Am J Respir Crit Care Med 157:17431747

5. Sood BG, Delaney-Black V, Aranda JV, Shankaran S 2004 Aerosolized PGE1: a selective pulmonary vasodilator in neonatal hypoxemic respiratory failure results of a phase I/II open label clinical trial. Pediatr Res 56:579-585

6. Sood BG, Peterson J, Malian M, Galli R, Geisor-Walter M, McKinnon J, Sharp J Maddipati KR 2007 Jet nebulization of prostaglandin E1 during neonatal mechanical ventilation: stability, emitted dose and aerosol particle size. Pharmacol Res 56:531541

7. O'Riordan TG, Palmer LB, Smaldone GC 1994 Aerosol deposition in mechanically ventilated patients. Optimizing nebulizer delivery. Am J Respir Crit Care Med 149:214-219

8. Dhand R 2004 Basic techniques for aerosol delivery during mechanical ventilation. Respir Care 49:611-622

9. Berthezene Y, Muhler A, Lang P, Shames DM, Clement O, Rosenau W, Kuwatsuru R, Brasch RC 1993 Safety aspects and pharmacokinetics of inhaled aerosolized gadolinium. J Magn Reson Imaging 3:125-130

10. Berthezene Y, Vexler V, Clement O, Muhler A, Moseley ME, Brasch RC 1992 Contrast-enhanced MR imaging of the lung: assessments of ventilation and perfusion. Radiology 183:667-672

11. Misselwitz B, Muhler A, Heinzelmann I, Bock JC, Weinmann HJ 1997 Magnetic resonance imaging of pulmonary ventilation. Initial experiences with a gadoliniumDTPA-based aerosol. Invest Radiol 32:797-801

12. Haage P, Adam G, Karaagac S, Pfeffer J, Glowinski A, Dohmen S, Gunther RW 2001 Mechanical delivery of aerosolized gadolinium-DTPA for pulmonary ventilation assessment in MR imaging. Invest Radiol 36:240-243

13. Haage P, Karaagac S, Adam G, Glowinski A, Gunther RW 2001 Comparison of aerosolized gadoteridol and gadopentetate dimeglumine for magnetic resonance ventilation imaging of the lung. Magn Reson Med 46:803-806

14. Haage P, Karaagac S, Adam G, Spuntrup E, Pfeffer J, Gunther RW 2002 Gadolinium containing contrast agents for pulmonary ventilation magnetic resonance imaging: preliminary results. Invest Radiol 37:120-125

15. Suga K, Ogasawara N, Okada M, Matsunaga N, Arai M 2002 Regional lung functional impairment in acute airway obstruction and pulmonary embolic dog models assessed with gadolinium-based aerosol ventilation and perfusion magnetic resonance imaging. Invest Radiol 37:281-291

16. Suga K, Ogasawara N, Tsukuda T, Matsunaga N 2002 Assessment of regional lung ventilation in dog lungs with Gd-DTPA aerosol ventilation MR imaging. Acta Radiol 43:282-291

17. Haage P, Karaagac S, Spuntrup E, Truong HT, Schmidt T, Gunther RW 2005 Feasibility of pulmonary ventilation visualization with aerosolized magnetic resonance contrast media. Invest Radiol 40:85-88

18. Nakagawa T, Sakuma H, Murashima S, Ishida N, Matsumura K, Takeda K 2001 Pulmonary ventilation-perfusion MR imaging in clinical patients. J Magn Reson Imaging 14:419-424

19. Walker GA 2002 Repeated measures analysis. In: Walker GA (ed) Common Statistical Methods for Clinical Research with SAS Examples. Cary, NC: SAS Institute Inc., pp 111-156

20. Stuart BO 1973 Deposition of inhaled aerosols. Arch Intern Med 131:60-73

21. Newman SP, Wilding IR 1999 Imaging techniques for assessing drug delivery in man. Pharm Sci Technolo Today 2:181-189

22. Kim CS 2000 Methods of calculating lung delivery and deposition of aerosol particles. Respir Care 45:695-711

23. Sangwan S, Condos R, Smaldone GC 2003 Lung deposition and respirable mass during wet nebulization. J Aerosol Med 16:379-386 
24. Dubus JC, Vecellio L, De Monte M, Fink JB, Grimbert D, Montharu J, Valat C, Behan N, Diot P 2005 Aerosol deposition in neonatal ventilation. Pediatr Res 58:10-14

25. Finlay WH, Stapleton KW, Chan HK, Zuberbuhler P, Gonda I 1996 Regional deposition of inhaled hygroscopic aerosols: in vivo SPECT compared with mathematical modeling. J Appl Physiol 81:374-383

26. Hatabu H, Chen Q, Stock KW, Gefter WB, Itoh H 1999 Fast magnetic resonance imaging of the lung. Eur J Radiol 29:114-132

27. Kauczor HU, Kreitner KF 1999 MRI of the pulmonary parenchyma. Eur Radiol 9:1755-1764

28. Attinger EO, Cahill JM 1960 Cardiopulmonary mechanics in anesthetized pigs and dogs. Am J Physiol 198:346-348
29. Truog WE, Gibson RL Jr, Henderson WR, Redding GJ 1990 Tumor necrosis factor-induced neonatal pulmonary hypertension: effects of dazmegrel pretreatment. Pediatr Res 27:466-471

30. Kauczor HU, Kreitner KF 2000 Contrast-enhanced MRI of the lung. Eur J Radiol 34:196-207

31. Montgomery AB, Paajanen H, Brasch RC, Murray JF 1987 Aerosolized gadolinium DTPA enhances the magnetic resonance signal of extravascular lung water. Invest Radiol 22:377-38

32. Kauczor HU, Hanke A, Van Beek EJ 2002 Assessment of lung ventilation by MR imaging: current status and future perspectives. Eur Radiol 12:1962-1970

33. Fink C, Ley S, Plathow C, Kauczor HU 2004 Ventilation imaging: proton MRI. Imaging Decisions MRI 8:24-28 